\title{
Avionics Box Cold Plate Damage Prevention
}

\author{
Lecture
}

\section{Abstract}

Over the years there have been several occurrences of damage to Space Shuttle Orbiter cold plates during removal and replacement of avionics boxes. Thus a process improvement team was put together to determine ways to prevent these kinds of damage. From this effort there were many solutions including, protective covers, training, and improved operations instructions. The focus of this paper is to explain the cold plate damage problem and the corrective actions for preventing future damage to aerospace avionics cold plate designs.

\section{Summary}

Thus this work will summarize the work that has been done to improve the human process for installing and removing avionics boxes on avionics shelves with cold plates for the Space Shuttle Orbiter. This work involves literature review of work performed at Kennedy Space Center for improving avionics box removal and installation, and discussions with expert operators and the process improvement team. The efforts from the process improvement team discovered several findings which resulted in many human factors related improvements. Although much was accomplished with this effort, there is still a need for better understanding the human capabilities for installing avionics boxes in restricted spaces such as in the avionics shelf.
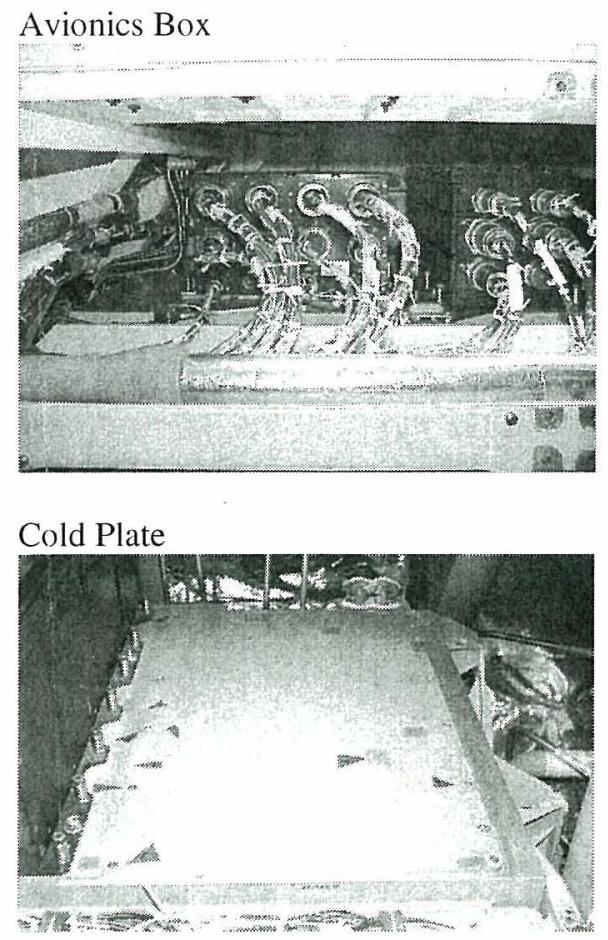


\title{
Avionics Box Cold Plate Damage Prevention
}

\author{
Damon B. Stambolian \\ NASA Kennedy Space Center (KSC) \\ Engineering and Technology Directorate \\ JFK Space Center \\ KSC, FL 32899 \\ 321-861-5973 \\ damon.b.stambolian@nasa.gov
}

List of co-authors: Steven W. Larchar, USA KSC; Gena Henderson, NASA KSC Engineering Directorate; and Donald Tran, NASA KSC Engineering Directorate; and Tim Barth, NASA Engineering and Safety Center (NESC)

\begin{abstract}
Over the years there have been several instances of damage to Space Shuttle Orbiter cold plates during removal and replacement of avionics boxes. Thus, a process improvement team was created to determine ways to prevent this kind of damage. From this effort there were many solutions including, protective covers, training, and improved operations instructions. The focus of this paper is to explain the cold plate damage problem and the corrective actions for preventing future damage to aerospace avionics cold plate designs.
\end{abstract}

\section{TABLE OF CONTENTS}

1. INTRODUCTION . .1

2. COLd Plates.

3. Process FaILURE MOdes ANd EFFECTS ANALYSIS ......3

4. METHODS FOR INSTALLING THE LRU

5. Cold Plate Damage Prevention

6. Mitigating Damage In Future DesignS..................... 6

7. CONCLUSION

REFERENCES.

ACKNOWLEDGEMENT.

BIOGRAPHY.

\section{INTRODUCTION}

The NASA Office of Inspector General (IG) had initiated a review of the Space Shuttle Cold Plate damage due to excessive handling. The number of cold plate problems had increased from an average of 16.5 per/year between 1990 through 2000 , to an average of 39.6 per year between 2001through 2005. The IG believed that the increase was indicative of handling problems and once the corrective actions in the IG report were instituted overall damages were reduced. [1]

Because of the significant increases in the number of Cold Plate and Line Replaceable Unit (LRU) damage, a Process Improvement Team (PIT) was formed to determine the cause of these damages, and to recommend corrective actions to mitigate future damages. The PIT recommended and implemented the following changes: standardization of cold plate mounted LRU removal and replacement (R\&R) procedures across multiple engineering disciplines, design and fabrication of cold plate covers to protect exposed surfaces, develop technician training and certification for cold plate mounted LRU R\&R and cold plate handling. Figure-1 is an example of the avionics boxes on shelves in the forward compartment of the Orbiter.

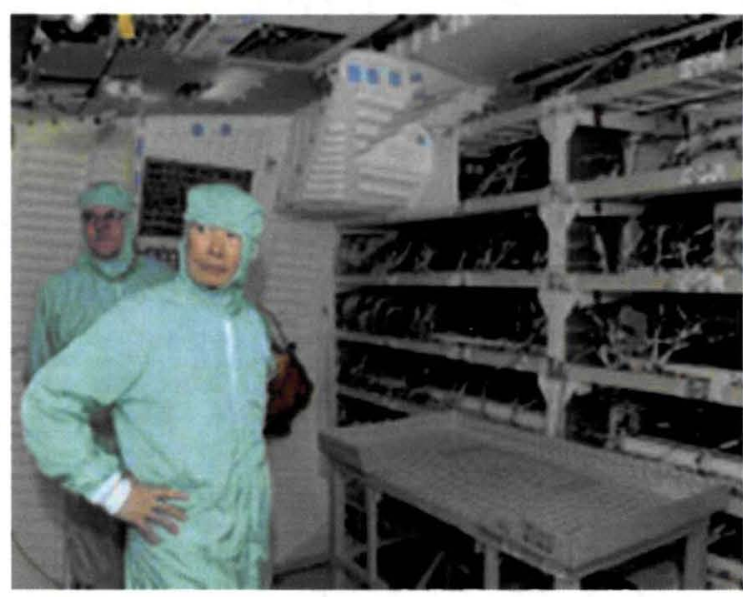

Figure 1 Avionics Boxes on Shelves in Forward Compartment with Star Trek Sulu (George Takei) visiting KSC.

\section{Cold Plates}

Cold plates dissipate heat from electronic components to ensure that the LRU does not overheat and fail or shorten the working life. Within the Space Shuttle Orbiters, cold plates are used in the forward and aft avionics compartments as well as the midbody. Cold water or Freon is pumped through the chambers inside the cold plate and through conduction heat is transferred from the LRU to the cold plate. The heat is then transferred out of the cold plate through cooling loops to heat exchangers and radiators on the orbiter. The constantly circulating water or Freon through the cold plates allows the avionics boxes to remain in constant operation without overheating. If a single cold plate fails, due to leakage, the loss of one of two coolant loops would result in mission termination. A damaged cold plate can seriously impact the schedule if detected, and 
become a safety concern if overlooked and a rupture occurs during flight. It is important to minimize the risk of damage.

Each cold plate is a precision engineered component that is expensive, time consuming to produce, and easily damaged. Each orbiter contains a set of 80 cold plates located in forward, mid, and aft compartments. Each complete set of 80 cold plates cost approximately $\$ 29$ million, an average of $\$ 362,500$ per cold plate. It takes four months to produce a single cold plate. Cold plates are produced using aluminum or stainless steel and vary in size and shape depending on its function. The top and bottom layers of the cold plate, called the upper and lower face sheets, are very thin. Because the face sheets are not much thicker than aluminum cans, the cold plates are very susceptible to damage. A cold plate may sustain damage during box installation, which may not be detectable because the box covers the damage. Cold plates are damaged primarily during the removal or installation of LRUs that are attached to the cold plate. The damage typically consists of scratches and small dents to the face sheet. See Figures (2-4)

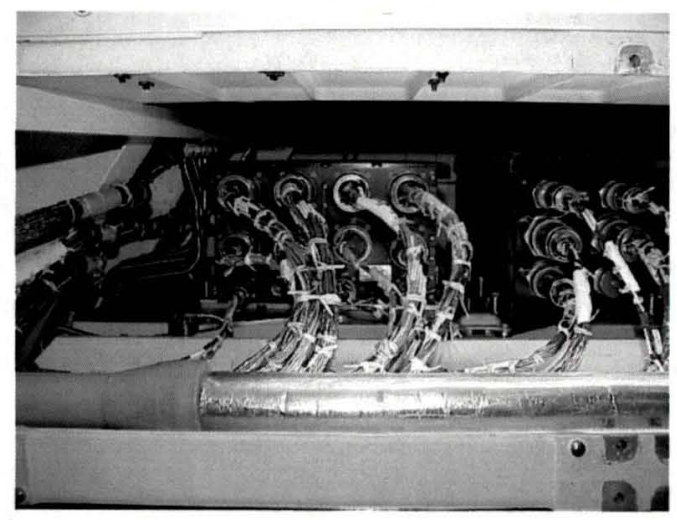

Figure 2 Avionics Box On Cold Plate

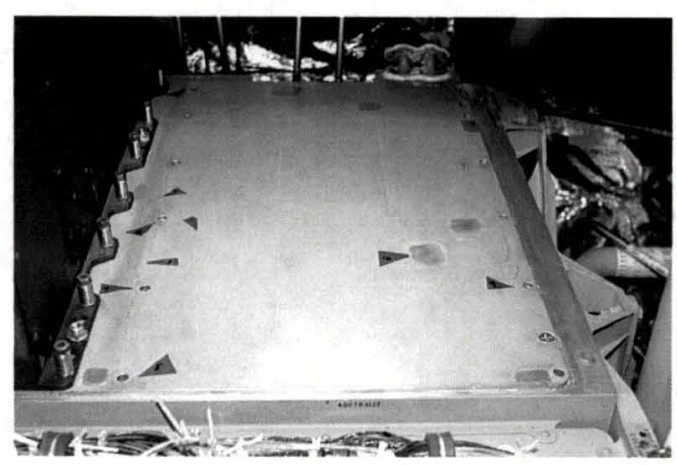

Figure 3 Cold Plate Without Avionics Box

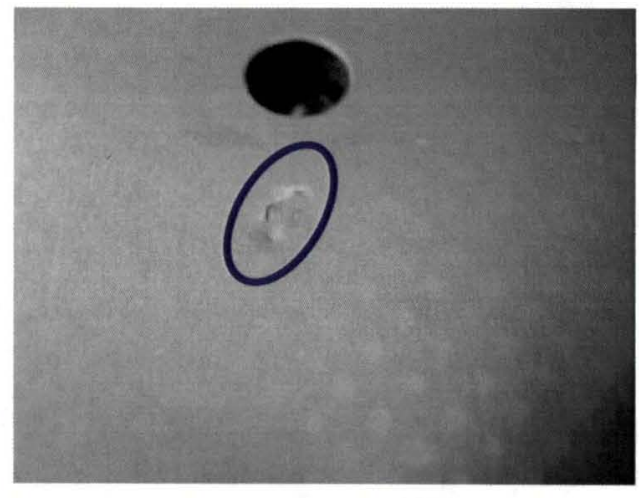

Figure 4 Close up of Cold Plate Damage

To access the actual damage entails making mold impressions of the damage and reading those impressions to determine the dimensions of the damaged area. The mold impression process begins with a quality inspector using mold material to create an inverse image of the actual damage on the cold plate (which is normally a dent, ding, or scratch). The mold material is pressed onto the cold plate over and around the damage area and the inspector applies light manual pressure to force the mold material into scratch, dent, or ding. The mold impression is analyzed and measured using a 3-dimensional optical analyzer. If the resultant calculated strain value of the cold plate damage exceeds the maximum strain levels, the cold plate will likely be scrapped; otherwise it will most likely be repaired and placed back in service.

Review of the Problem Report and Corrective Action (PRACA) database found 379 PRACA reports that described at least one instance of cold plate damage from 1990 through December 7, 2005. Many of these reports noted multiple instances of damage in a single PRACA report. Analysis of this data showed a substantial increase in reports of cold plate damage beginning in 2001. From 1990 through 2000, there were 181 PRACA reports that included at least one instance of cold plate damage. From 2001 through 2005, there were 198 PRACA reports which noted at least one instance of cold plate damage. The annual average number of PRACA reports of cold plate damage increased from 16.5 for 1990-2000 to 39.6 for 2001-2005. For example, between 2001 and 2003, 11 cold plates, valued at $\$ 2.79$ million, were damaged so badly that they were deemed usable and were scrapped. This led to the addition of government inspectors to observe the removal and installation process. This would allow NASA to determine and document whether cold plate damage was pre-existing or occurred during shuttle processing at KSC. By establishing when, where, and how the cold plate damage occurred, process improvements could be implemented to prevent damage by addressing the root causes for cold plate problems. 
Additional mitigation actions to minimize cold plate damage were taken. Protective covers have been fabricated to protect the cold plates while the LRU is not installed. Engineering conducts a pre-task briefing prior to each LRU installation or removal. These briefings are to ensure that Shop, Quality, and Engineering personnel have reviewed the procedure and understand the instructions. Engineering will be present for all LRU installations.

\section{Process Failure Modes and Effects ANALYSIS}

A Process Failure Modes and Effects Analysis (PFMEA) was performed for the removal and installation procedures impacting the orbiter Environmental Control and Life Support Systems (ECLSS) cold plates. Procedures analyzed in this process include orbiter black box removal and installation.

A step-by-step analysis was performed for the black box removal and installation procedures that utilized different methods. These methods included Ground Support Equipment (GSE) tracks and rollers, air bags, manual lifting, and the rope method.

For each of these procedures, a detailed analysis was performed identifying the constituent tasks and possible damage to orbiter cold plates during execution of the tasks. An assumption was made by the team that all the cold plate damage is detectable. This resulted in the generation of failure mode consequences, likelihood, current mitigating procedures/practices, and recommended follow-on actions.

It was determined the highest risk areas for damaging cold plates were associated with LRU installation and removal. The following identifies the most significant areas of concerns and underlying causes during this evaluation:

- $\quad$ LRU Fastener Installation/Removal: The remote torque tool slips off fastener and contacts the cold plate. Difficulty aligning Blind Fasteners to cold plate holes can cause fastener to contact with cold plate face sheet.

- GSE Track and Roller Bar Installation/Removal: Remote torque tool slips off roller bar fastener and contacts cold plate Track contacts cold plate during installation and removal.

- $\quad$ LRU contacts cold plate

The short term and long term recommended actions were identified. Short term resolutions will be implemented by procedural controls.

- Add/verify shim stock protection installed between LRU and cold plate.

- Add foam protection to all exposed cold plate surfaces during LRU installation and removal (specifically between LRU boxes).

- Require the use of female torque tool during manual LRU installation/removal

- Specify the use of three technicians while installing or removing LRU manually.

As a result, a Process Improvement Team (PIT) was chartered to resolve cold plate processing concerns including damage, and to address all recommended actions (short and long term) generated from the PFMEA.

The PIT evaluated over 50 problems or potential problems regarding cold plate mounted LRU R\&R. Proposed solutions were categorized, and as a result specific recommendations were proposed. The recommendations were to:

- Enhance procedures for LRU and cold plate R\&R to reduce potential for cold plate damage.

- Standardize tasks across LRU engineering disciplines.

- Provide nearly $100 \%$ protection of exposed cold plate surfaces while LRU is removed.

- Heighten cold plate training and awareness.

- Develop LRU R\&R certification

- Dedicated LRU R\&R team until shop personnel certified.

- Task team briefings prior to LRU R\&R

\section{Methods FOR InSTALling The LRU}

Although there are several areas that can lead to damage to cold plates, such as use of tools, removing cables, etc., this paper will mainly focus on its R\&R of LRU.

For preparing $R \& R$ access the technicians will insure that all obstructions including brackets are removed. Cable clamps and spot ties are removed as required. Wires are moved out of the way so that they do not violate the specified bend radius on wires and harnesses. The area in front of an LRU opening should include protection for wire harnesses that may be in front of the LRU.

Once access has been determined, the following methods are used for R\&R of the LRU.

\section{Non Mechanically Assisted Manual Installation}

The non-mechanically assisted manual lift installation is simple but the potential for cold plate damage exists if the LRU is not lifted cleanly or installed cleanly. Because no mechanical aid is used, this method should only be used on lightweight LRUs mounted on the top of the shelf, such as MDMs which are about 36 pounds. See Figure-5 


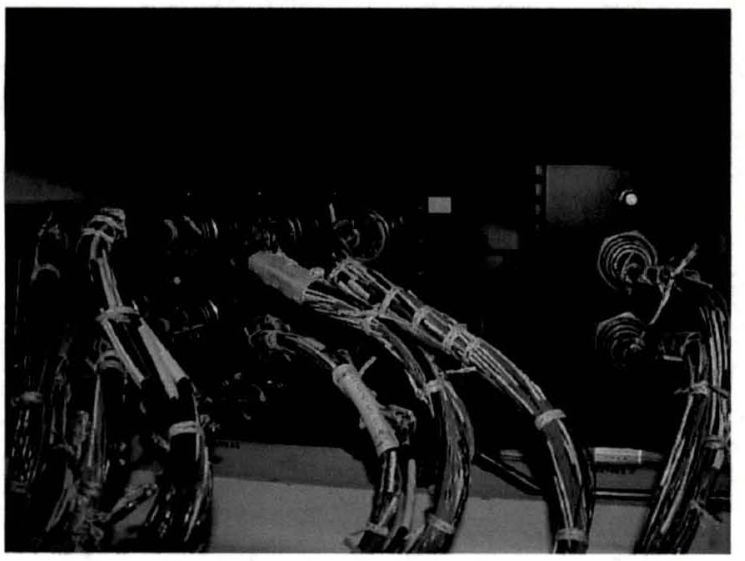

Figure 5 Example of Box for Non-Assisted Method

There is a detailed Operations Maintenance Instruction (OMI) that provides the detailed work steps for the technician to remove and/or install an LRU from an orbiter cold plate using manual methods. It does include a step to inspect the removed LRU. It does not include other areas that may lead to damage to cold plates.

The OMI gives instructions to inspect the mounting location to verify the manual technique will work in the current vehicle and avionics bay configuration per the following. Confirm the LRU is accessible and free from obstruction that would interfere with a technician's manual ability to lift and/or position the LRU. There are caution labels in the work steps to record if there are LRUs adjacent to the LRU being removed and if the LRUs are installed or not. And if the removal of this LRU is not followed immediately by an installation then apply the appropriate cold plate protective cover (Figure-10). Other caution labels explain that the cold plate LRU mounting surface is very thin and is very easily damaged and instruct the technician that failure to follow the prescribed restrictions may cause irreparable damage to the cold plate. The following are recommendations to prevent damage.

- Do not slide the LRU on the Cold plate surface or allow LRU corners to nick, gouge, scratch or ding cold plate.

- Use proper protective covers to aid and protect during LRU removal.

- Make sure the LRU does not tilt or slide during the removal.

- Inspect the LRU mounting location to verify the manual technique will work in the current vehicle and avionics bay configuration to confirm the LRU position is accessible and free of obstructions that would interfere with a technician's manual ability to lift and/or position the LRU.

- Make sure the LRU does not tilt or slide during the installation, which may result in cold plate damage.
- Locate the LRU to the mounting location allowing the LRU to gently contact the shim-stock sandwich material protecting the cold plate surface.

- Make small corrections to align the LRU captive fasteners with the mounting holes.

\section{Track and Roller Bar Installation}

The track and roller bar installation method aids the technician to guide the avionics box over the cold plate, and then to mechanically lower the box onto the plate. Tracks and Roller bars do not work in all locations on all vehicles. Slight differences in construction will allow use in some vehicles but not others for the same slots. They are bulky and awkward to use, pose a significant risk to damage cold plate during GSE installation. Tracks and Roller bars often cause damage to wires and other interferences on the orbiter. See Figures 6 and 7 for track and roller bar.



Figure 6 Track and Roller Bar Hardware



Figure 7 Track and Roller Bar Drawing with Box

The technician is not required to use of Tracks and Roller Bars. The specification already has several LRUs that are 
exempt from track and roller bars because this method does not work for that LRU. The rail set is provided to aid the installation and removal of avionics LRUs and to prevent LRU and cold plate damage during handling.

The GSE shall be utilized for LRU installation and removal when required by installation drawing unless use is specifically excluded. There are functional or operational limitations that warrant an alternate approach. Any alternate method utilized will be the responsibility of the user and must incorporate a means for safe handling as well as protection for both cold plate and LRU mating surfaces. This allows the technician to use whatever method they deem necessary if there is some limitation to their use.

The cold plate PIT team found no evidence that any one method had caused more damage than another. However, there are recorded incidents of the alignment pin and bracket at the end of the track causing damage. The same for the male torque tool slipping and impacting the cold plate. In fact the use of tracks and roller bars necessitates the use of the male Allen head wrench remote torque tool. In many cases the roller bars must be partially disassembled. Because of their size and awkwardness they may cause incidental damage to wiring and ECL lines.

\section{Airbag Assisted Installation}

The airbag method utilizes an inner tube enclosed in a bag along with associated hardware. The box is still manually lifted into the opening, but the airbag aids in lower/lifting the box to the cold plate. This method is only useful on LRUs that hang under the shelf. The Airbag must be checked before use for operation and lifting capability. The airbag is inflated to lift the LRU to the receiving cold plate surface during installation, and deflated to lower the LRU away from the cold plate during removal. The potential causes of damage are uneven contact of the LRU to the cold plate if the LRU mating surface does not remain parallel with the cold plate during lifting, and incidental damage to flight hardware that supports the weight of the LRU on the airbag. Unexpected LRU movement during fastener removal is another potential cause of damage. The air bag should fully support the LRU. See Figure-8

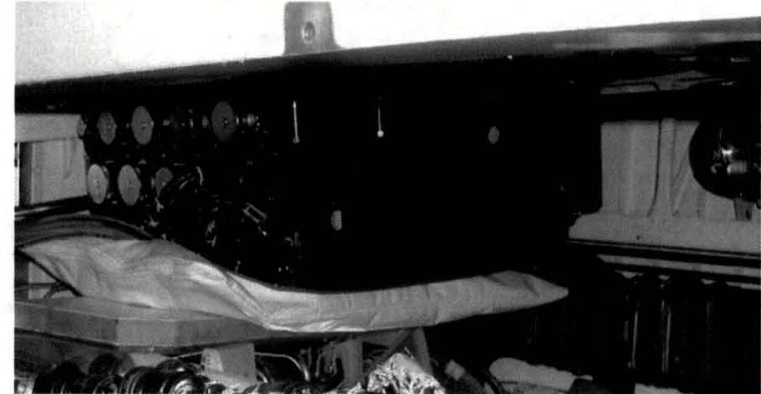

Figure 8 Air Bag Method

\section{Rope Assisted Method}

This method uses a Rope to install/remove an LRU out of a bay for vertically mounted cold plates. Most risky of all methods, potential causes of damage are from the LRU swinging and making contact with the cold plate. This method requires three people to perform to make sure that the LRU is controlled at all times during the process. The LRU is essentially removed by pulling it out with a Rope. One technician lifts with the rope while the other two guide the LRU to prevent damage. See Figure-9 for vertical installation of LRU.



Figure 9 Vertical Method

\section{Cold Plate Damage Prevention}

The cold plate damage prevention program includes; damage mitigation techniques, new certifications, for cold plate mounted LRU R\&R; and cold plate handling, as well as enhanced training, and awareness. 


\section{Damage Mitigation}

New OMI's have been developed such that all disciplines that author cold plate mounted LRU R\&R procedures use these new procedures for consistency regarding added cold plate protection techniques. These new procedures address the four methods of LRU R\&R. These procedures include steps for engineering to conduct a Pre-Task Briefing prior to each LRU installation or removal. These briefings are to ensure that the all disciplines (Shop, Quality, and Engineering) have reviewed the WADs, agree on the proper installation method, and understand the instructions. The steps also include that engineering is present for all first time LRU installations, and that the protective covers are used for all vehicles (See Figure-10).

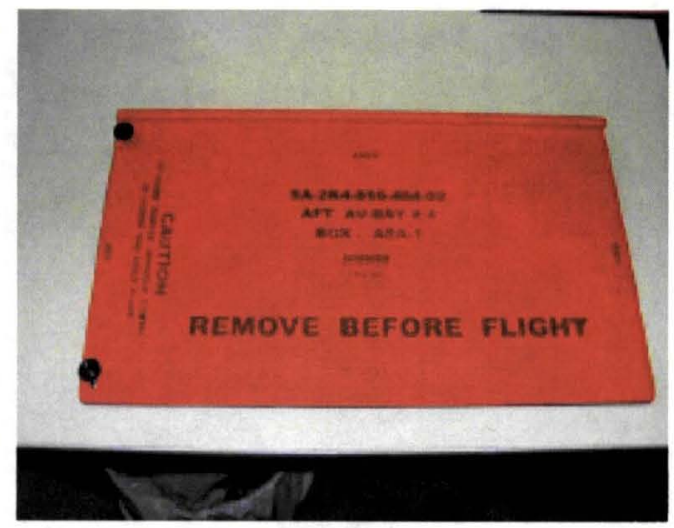

Figure 10 Cold Plate Cover

\section{Certifications}

There are two new certifications created to ensure that personnel have been adequately trained and have had experience in performing Cold Plate operations. The Certifications are 1) Cold Plate Mounted LRU R\&R 2) Cold Plate Handling

\section{Training}

Two new classes were developed to support this effort to reduce damage to cold plates. 1) Cold Plate Familiarization: is computer based training format. It is required for all personnel working in and around cold plates. The primary focus is to provide education on the susceptibility of cold plates to damage, ensure that surface protection is in place and prevent surface contact. 2) Cold Plate Handling and Cold Plate Mounted LRU Removal and Replacement: This course is an instructor lead class that will cover in detail all aspects of cold plate handling and LRU R\&R. There is a "hands on" component of this class, with avionics bay mock-ups for practicing LRU R\&R, and actual cold plates for demonstrations.

\section{Mitigating Damage In Future Designs}

The cold plates made with stainless steel seem to better withstand the rigors of $R \& R$. Numerous Work Authorization Documents (WAD) have been generated due to the damage done to cold plates during system R\&R. New design utilizing the same stainless steel hardened material for cold plates throughout the vehicle could be used in the future. Or a harder aluminum grade could be used than what was used in the Orbiter. Since weight savings is a premium, future cold plates will likely be constructed of aluminum. This could reduce risk to collateral damage to the cold plates, and thus decrease the time spent on repairing cold plates, as well as the number of operational hits to the schedule while waiting for repairs to be completed. [2]

During the Constellation program analysis was done for the Ares rocket to improve avionics box placement for the technicians. [3] In order to have the efficient and effective ground processing inside and outside the vehicle, all of the ground processing activities were analyzed. The analysis was performed, by engineers, technicians, and human factors experts with spacecraft processing experience. The procedure used to gather data was accomplished by observing human activities within physical mockups. See Figure-11

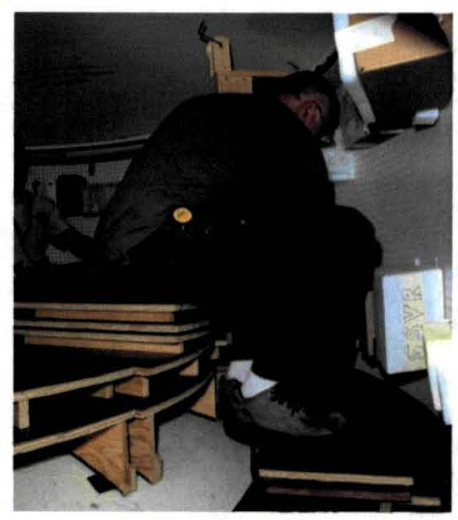

Figure 11 Designing Box Locations and Ground Support Equipment

Most recently for the Orion vehicle motion capture is being used to design the avionics box and cold plate configuration for boxes lowered into the floor. $[4,5]$ The computer models include the CAD flight hardware and human Avatars. The envelope spaces between the human the avionics shelf and the box and avionics shelf can be viewed and the stresses to the human can be determined. These kinds of studies help us 
to determine the best design solution for installing the boxes. See Figure-12



Figure 12 Recent Study on Orion Avionics Boxes

Still, more needs to be done to understand the basic human factor capabilities for installing boxes carefully in restricted spaces so we can design properly for these activities. One recent study at the University of Miami is exploring this area by simulating the avionics box and avionics shelf configuration in a biomechanics laboratory. [6] This study looked at lifting time, how close the box can be placed on target, the EMG muscle activity, and the forces to the L5/S1. The lifts were manually done with restrictions or no restrictions to the installed box-with three different box weights, and two shelf heights. See Figure-13


Figure 13 Recent University Study

\section{Conclusion}

Much was learned during the Shuttle Program about how cold plate damages occur during R\&R of avionics boxes. Although many procedural and barrier improvements were made to mitigate damages this with the already designed avionics box and shelves, there are still occurrences of cold plate damage. More work needs to be done to understand the humans ability for installing and removing avionics boxes, and to use this knowledge towards proactive designs during future programs which use avionics box/shelf and cold plate configurations.

\section{REFERENCES}

[1] Office of Inspector General. Washington, DC. Final Memorandum on the Review of Space Shuttle Cold Plates (Report No. IG-06-012; Assignment No. S-06-004) April 28, 2006

[2]NASA Lesson Learned Entry: 3696. 2010. Avionics Cooling. https://nen.nasa.gov/web/ll/home/llis-doc-

viwer?url=https://nen.nasa.gov/lis_content/imported_content/less on $3696 . \mathrm{html}$

[3] Gregory M. Dippolito \& Damon B. Stambolian. Co-authors; Bao Nguyen, Charles Dischinger, Donald Tran, Gena Henderson, Dr. Tim Barth. Human Factors Analysis to Improve the Processing of Ares-1 Launch Vehicle. 2011 IEEEACpaper\#1022

[4] Damon B. Stambolian., Marie-Jeanne O. Steady Ndiaye., Brad A. Lawrence., Katrine S Stelges., Lora C. Ridgwell., Mary K Osterhout., Robert E. Mills., Gena Henderson., Donald Tran., and Tim Barth. Human Modeling for Ground Processing Human Factors Engineering Analysis. 2012 IEEEACpaper\#0175

[5] Jeffrey S. Osterlund \& Brad A. Lawrence. 61st Virtual Reality: Avatars in Human Spaceflight Training. International Astronautical Congress, Prague, CZ. Copyright (C2010 by the International Astronautical Federation.

[6] Damon B. Stambolian, Shihab S. Asfour, Moataz Eltoukhy, and Stephanie Bonin. Avionics Box Precision Placement in Restricted Space. XXIIIrd Annual International Occupational Ergonomics and Safety Conference. June 9-10, 2011

\section{ACKNOWLedgement}

This acknowledgment is for Carol Anne Dunn for reviewing the paper to improve its readability.

\section{BIOGRAPHY}



Damon Stambolian is currently Engineering focusing his research on Biomechanics at the University of Miami's Biomechanics Laboratory. He is also currently working in the KSC Engineering and Technology Directorate at Kennedy Space Center. Prior to working in the Engineering Directorate, he worked in; the Constellation Ground Operations Project office, the Space Station Program within the Orbiter Space Plane Project at KSC, and the Space Shuttle Program at KSC. Within these Programs, he was involved with human factors related process improvements for ground processing operations, i.e., assembly, maintenance, inspection of flight hardware. 